\title{
Predictors of a Cerebrovascular Accident in a Population of Systemic Sclerosis Patients Followed at a Large Academic Center with a Dedicated Scleroderma Center
}

\author{
Christopher J. Inserra1 ${ }^{(0)}$, Chris T. Derk ${ }^{2 *}$ (]) \\ ${ }^{1}$ Department of Medicine, Creighton University, Omaha, NE, USA \\ ${ }^{2}$ Division of Rheumatology, University of Pennsylvania, Philadelphia, PA, USA \\ Email: ${ }^{\star}$ Chris.Derk@pennmedicine.upenn.edu
}

How to cite this paper: Inserra, C.J. and Derk, C.T. (2020) Predictors of a Cerebrovascular Accident in a Population of Systemic Sclerosis Patients Followed at a Large Academic Center with a Dedicated Scleroderma Center. Open Journal of Rheumatology and Autoimmune Diseases, 10, 45-56. https://doi.org/10.4236/ojra.2020.102006

Received: March 11, 2020

Accepted: April 7, 2020

Published: April 10, 2020

Copyright $\odot 2020$ by author(s) and Scientific Research Publishing Inc. This work is licensed under the Creative Commons Attribution International License (CC BY 4.0).

http://creativecommons.org/licenses/by/4.0/

\begin{abstract}
Aim: The aim of this study is to describe the clinical characteristics of patients with a diagnosis of systemic sclerosis who later suffer a stroke and to identify associations for this relationship. Background: Prior studies have showed an increased risk of cardiovascular disease among patients with chronic inflammatory disorders, with chronic inflammation leading to atherosclerosis believed to be the culprit. Systemic sclerosis (SSc) is a chronic inflammatory disease characterized by diffuse fibrosis of the skin and internal organs. Previous studies have suggested a possible link between systemic sclerosis and macrovascular complications such as stroke. Methods: This is a retrospective chart review of patients treated within the University of Pennsylvania Health System from October 2015 to April 2019 with a diagnosis of SSc. Using ICD10 codes, we identified a cohort of SSc patients who suffered a stroke. Information regarding demographics and stroke risk factors were gathered from the charts of patients with a diagnosis of both SSc plus stroke and compared to a control group of randomly selected patients with SSc who never suffered a stroke. Continuous variables were conveyed using a mean plus a standard deviation. A two-sample $t$-test was used to compare the two groups of patients. Qualitative variables were compared using a two-tailed Fisher's exact test. Results: Based on a large cohort of SSc patients $(n=2080)$ followed between October 2015 and April 2019, we identified 36 SSc patients who developed a subsequent stroke ( $1.7 \%$ of cohort). When looking at risk factors for stroke in SSc patients, we identified hypertension and atrial fibrillation to be associated with the diagnosis of stroke in such patients. Specifically, 28 of the 36 patients with both SSc and stroke also had a diagnosis of
\end{abstract}


hypertension while in the control group, only 17 of 36 patients had hypertension. Atrial fibrillation was seen in 9 of 36 patients with both SSc and CVA while it was seen in only 2 of 36 patients in the control group. Conclusions: This case control study demonstrated that the presence of hypertension and atrial fibrillation had a statistically significant association with the diagnosis of CVA in patients with SSc.

\section{Keywords}

Systemic Sclerosis, Scleroderma, Stroke, Cerebrovascular Accident, Atrial Fibrillation, Hypertension

\section{Background}

Systemic sclerosis (SSc) is a chronic autoimmune rheumatic disorder characterized by both cellular and humoral immune dysregulation, excessive extracellular matrix and collagen deposition in the skin and internal organs, and microvasculature dysregulation. It has been shown that there is an increased risk for cardiovascular disease among patients with other chronic autoimmune rheumatic disorders, with chronic inflammation leading to atherosclerosis believed to be the culprit [1]. For example, elevated systemic inflammatory markers have been reported to result in an increased risk for cardiovascular death in patients with rheumatoid arthritis even when controlling for traditional cardiovascular comorbidities and risk factors [2]. In addition, patients with psoriatic arthritis have been shown to have increased severity, prevalence, and burden of coronary atherosclerosis when imaged with coronary computed tomography angiography [3].

From a pathophysiological standpoint, inflammation incites the development of atherosclerotic plaques and also contributes to the degradation of their overlying fibrous caps, thus contributing to plaque rupture and subsequent coronary artery infarction [4]. While the exact process remains uncertain, chronic inflammation may stimulate the expression of adhesion molecules (such as vascular adhesion molecule-1, intercellular adhesion molecule-1, and E-selectin), cause endothelial dysfunction, induce smooth muscle cell survival, migration, and proliferation in the arterial intima, and then consequently lead to atherosclerotic plaque formation. Proinflammatory cytokines may also influence the release of matrix metalloproteinases, thus disrupting the balance of elastin and collagen while increasing plaque vulnerability through plaque remodeling and degradation of the fibrous cap [5].

While microvasculature involvement of small arterioles and capillaries is more commonly associated with SSc, the macrovascular complications of coronary artery disease (CAD) and myocardial infarction have been thought to be linked to SSc as well. In fact, coronary artery vasospastic events ("Raynaud's phenomenon") in patients with SSc have been suggested to result in myocardial ische- 
mia, symptoms of angina, and reperfusion injuries; ultimately leading to myocardial fibrosis. Because normal-appearing coronary arteries are more commonly seen in SSc patients who suffer acute myocardial infarction than in the general population, it has been hypothesized that the attraction of fibroblasts into coronary artery walls and their ultimate transition into myofibroblasts which produce increased amounts of extracellular matrix proteins results in increased intimal wall thickness and the aforementioned vasospastic instability. Such changes in intimal wall thickness predispose to endothelial injury, thus allowing exposed subendothelium to contribute to platelet aggregation and thrombus formation [6] [7].

Cerebrovascular accidents (CVA), can be either ischemic or hemorrhagic and are major causes of death and morbidity worldwide [8]. The most common cause of hemorrhagic stroke is hypertensive small-vessel disease, which causes small aneurysms that eventually rupture [9]. Approximately $80 \%$ of all CVAs are ischemic, defined by some form of vessel occlusion [10]. Chronic inflammation related to autoimmune rheumatic diseases is known to result in a hypercoagulable state given the fact that inflammatory cytokines have been shown to induce the coagulation cascade, downregulate the anti-coagulation pathway, and inhibit fibrinolysis [11]. Vasculitis is a disease state defined by inflammatory infiltrates in the vessel wall leading to narrowing of the vessel lumina and a subsequent increased risk [12]. Similarly, systemic lupus erythematosus is a chronic systemic inflammatory disease where individuals have twice the risk of developing ischemic stroke compared to the general population [13].

Few studies have examined the relationship between SSc and stroke. Two epidemiological studies saw an increased risk of ischemic stroke in patients with SSc [14] [15] while a third evaluated the risk but did not find it statistically significant [16]. A more recent meta-analysis revealed a statistically significant increase of ischemic stroke risk in patients with SSc [17]. To further investigate this relationship, we designed a retrospective case control study to define predictors for the development of a CVA in patients with SSc based on a case control model.

\section{Methods}

This is a retrospective chart review of patients treated within the University of Pennsylvania Health System (UPHS) from October 2015 to April 2019 with a diagnosis of SSc. We identified a cohort of SSc patients who suffered a CVA. All patients were diagnosed with one of the ICD10 codes for SSc (M34, M34.0, M34.1, M34.2, M34.8, M34.81, M34.9). The ICD10 code I63.9 was used to identify patients diagnosed with a CVA. The ICD10 coding system was launched at UPHS in October 2015; ICD9 codes were not used in this study. Information regarding demographics and CVA risk factors were gathered from the charts of patients with a diagnosis of both SSc plus CVA and compared to a control group of randomly selected patients with SSc who never suffered a CVA. The control 
group was selected by randomization which was performed using a computerized random-number generator of the patients selected who did not have a CVA. Patient demographics that were recorded included sex, race, age at diagnosis of most recent CVA, and age at diagnosis of SSc. Variables collected included: presence of hypertension, dyslipidemia, prior transient ischemic attack (TIA) or CVA, CAD, atrial fibrillation, diabetes, tobacco use, family history of CVA, carotid disease, type of SSc, anti-nuclear antibodies (ANA), Scl-70 antibodies, anti-centromere antibodies (ACA), serum creatinine at time of most recent CVA, hemoglobin at time of most recent CVA, and serum albumin at time of most recent CVA.

Continuous variables were conveyed using a mean plus a standard deviation. A two-sample $t$-test was used to compare the two groups of patients. Qualitative variables were compared using a two-tailed Fisher's exact test. A level of $\alpha=0.05$ was used to demarcate statistical significance.

\section{Results}

From 2080 SSc patients actively followed within the University of Pennsylvania Health System from October 2015 to April 2019, 36 (1.7\% of the actively followed SSc population) patients were diagnosed with a CVA following their diagnosis of SSc. Originally, 51 patients were identified as having been diagnosed with one of the aforementioned ICD10 codes for SSc + the ICD10 code for CVA. However, after reviewing the charts of these patients, a total of 15 were excluded because they met one of the following criteria: 1) no clear diagnosis of CVA or SSc after thorough chart review or 2) lack of information regarding CVA risk factors after thorough chart review. One patient out of the 15 was excluded because she was diagnosed with a CVA as a newborn. Thirty-six SSc patients who never suffered a CVA were then randomly selected as the control population with the aid of a computerized random-number generator. Patients with a CVA were diagnosed with SSc at a younger age $(51.8 \pm 15.7$ years of age, $n=31)$ compared to those who were not diagnosed with a CVA $(52.6 \pm 14.7$ years of age, $n=36$ ) though this difference was not statistically significant (Table 1). Cerebrovascular accident diagnosis occurred at a mean age of $60.8 \pm 13.6(n=35)$ years of age. If a patient was diagnosed with more than one CVA, the age at diagnosis of their most recent CVA was used. Both groups consisted of substantially more females than males, although there was not a statistically significant difference in the ratio of females to males between the groups.

Hypertension and atrial fibrillation were more commonly seen in patients who suffered a CVA and these differences were statistically significant. Specifically, 28 of the 36 patients with SSc + CVA had hypertension while only 17 of the 36 patients with SSc but no CVA had hypertension $(p=0.014)$. Similarly, nine of the 36 patients with SSc + CVA had atrial fibrillation while only two of the 36 patients with SSc but no CVA had atrial fibrillation $(\mathrm{p}=0.046)$. Other common comorbidities for a CVA such as hyperlipidemia, diabetes mellitus, to- 
bacco use, family history of CVA, and carotid artery disease were also more commonly seen in patients who suffered a CVA though the differences for these parameters between the two groups were not statistically significant. Coronary artery disease was very close to reaching statistical significance though likely did not due to our small sample size. In both groups, the limited form of systemic sclerosis was more common, although there was not a statistically significant difference in the ratio of diffuse SSc to limited SSc between the two groups. Of the $35 \mathrm{SSc}$ patients whose age was known at diagnosis of their CVA, nine of them had a prior transient ischemic attack or CVA. At the time of CVA diagnosis, our SSC cohort had a mean serum creatinine of $1.23 \pm 0.77 \mathrm{mg} / \mathrm{dl}(\mathrm{n}=26)$, a mean hemoglobin of $12.0 \pm 2.21 \mathrm{gm} / \mathrm{dl}(\mathrm{n}=26)$, and a mean serum albumin of $3.7 \pm 0.53 \mathrm{gm} / \mathrm{dl}(\mathrm{n}=24)$.

Table 1. Comparison of SSc patients with a CVA to SSc patients without a CVA.

\begin{tabular}{|c|c|c|c|}
\hline Parameter & $\mathrm{SSc}+\operatorname{CVA}(\mathrm{n}=36)$ & $\mathrm{SSc}+$ no CVA $(\mathrm{n}=36)$ & $p$ value \\
\hline Sex & $6 \mathrm{M} / 30 \mathrm{~F}(\mathrm{n}=36)$ & $3 \mathrm{M} / 33 \mathrm{~F}(\mathrm{n}=36)$ & 0.48 \\
\hline Race & $14 \mathrm{AA} / 20 \mathrm{C} / 2 \mathrm{H}(\mathrm{n}=36)$ & $6 \mathrm{AA} / 29 \mathrm{C} / 1 \mathrm{H}(\mathrm{n}=36)$ & N/A \\
\hline Age at CVA dx & $60.8 \pm 13.6(n=35)$ & N/A & N/A \\
\hline Age at SSc dx & $51.8 \pm 15.7(\mathrm{n}=31)$ & $52.6 \pm 14.7(\mathrm{n}=36)$ & 0.83 \\
\hline Hypertension & 28 yes $/ 8$ no $(n=36)$ & 17 yes/19 no $(n=36)$ & 0.014 \\
\hline Hyperlipidemia & 20 yes/16 no $(n=36)$ & 16 yes $/ 20$ no $(n=36)$ & 0.48 \\
\hline Prior TIA or CVA & 9 yes/26 no $(n=35)$ & $\mathrm{N} / \mathrm{A}$ & $\mathrm{N} / \mathrm{A}$ \\
\hline CAD & 10 yes/26 no $(n=36)$ & 3 yes/33 no $(n=36)$ & 0.06 \\
\hline Atrial fibrillation & 9 yes $/ 27$ no $(n=36)$ & 2 yes/34 no $(n=36)$ & 0.046 \\
\hline Diabetes mellitus & 6 yes $/ 30$ no $(n=36)$ & 3 yes/33 no $(n=36)$ & 0.48 \\
\hline Tobacco use & 18 yes/18 no $(\mathrm{n}=36)$ & 15 yes/21 no $(\mathrm{n}=36)$ & 0.64 \\
\hline Family hx of CVA & 6 yes/29 no $(n=35)$ & 4 yes/32 no $(n=36)$ & 0.51 \\
\hline Carotid disease & 13 yes/13 no $(n=36)$ & 2 yes/1 no $(n=3)$ & 1 \\
\hline Type of SSc & $6 \mathrm{D} / 19 \mathrm{~L}(\mathrm{n}=25)$ & $7 \mathrm{D} / 29 \mathrm{~L}(\mathrm{n}=36)$ & 0.76 \\
\hline Anti-nuclear antibody & $24 \mathrm{pos} / 1$ neg $(\mathrm{n}=25)$ & $32 \mathrm{pos} / 3$ neg $(\mathrm{n}=35)$ & 0.63 \\
\hline Scl-70 antibody & $1 \mathrm{pos} / 16$ neg $(\mathrm{n}=17)$ & $4 \operatorname{pos} / 26$ neg $(n=30)$ & 0.64 \\
\hline $\mathrm{ACA}$ & 10 pos $/ 14$ neg $(n=24)$ & 17 pos/16 neg $(n=33)$ & 0.59 \\
\hline Cr at time of CVA & $1.23 \pm 0.77 \mathrm{mg} / \mathrm{dl}(\mathrm{n}=26)$ & N/A & N/A \\
\hline $\mathrm{Hb}$ at time of CVA & $12.0 \pm 2.21 \mathrm{gm} / \mathrm{dl}(\mathrm{n}=26)$ & N/A & N/A \\
\hline Alb at time of CVA & $3.7 \pm 0.53 \mathrm{gm} / \mathrm{dl}(\mathrm{n}=24)$ & $\mathrm{N} / \mathrm{A}$ & $\mathrm{N} / \mathrm{A}$ \\
\hline
\end{tabular}

$S S c$ systemic sclerosis, $C V A$ cerebrovascular accident, $M$ male, $F$ female, $A A$ African American, $C$ Caucasian, $H$ Hispanic, $N / A$ not applicable, $d x$ diagnosis, $T I A$ transient ischemic attack, $C A D$ coronary artery disease, $h x$ history, $D$ diffuse, $L$ limited, pos positive, neg negative, $A C A$ anti-centromere antibody, $C r$ creatinine, $H b$ hemoglobin, $A l b$ albumin. 
We then did a subgroup analysis based on type of SSc, comparing patients with DcSSc + CVA versus DcSSc with no CVA as well as LcSSc + CVA versus LcSSc with no CVA. Out of the 36 patients in the SSc + CVA group, only 25 had a clear definition of type of SSc. In the first subgroup analysis of DcSSc patients, none of the parameters were statistically significant although CAD was close (Table 2).

In our comparison of LcSSc + CVA versus LcSSc with no CVA, we found a statistically significant difference with regards to race with more African American patients having LcSSc + CVA than LcSSc with no CVA. Specifically, eight of the 19 patients with LcSSc + CVA were African American while only four of the 29 patients with LcSSc but no CVA were African American ( $p=0.041)$. The rest of the parameters did not reach statistical significance (Table 3 ).

Table 2. Comparison of DcSSc patients with CVA to DcSSc patients without a CVA.

\begin{tabular}{|c|c|c|c|}
\hline Parameter & $\operatorname{DcSSc}+\operatorname{CVA}(n=6)$ & $\mathrm{DcSSc}+$ no CVA $(\mathrm{n}=7)$ & $p$ value \\
\hline Sex & $1 \mathrm{M} / 5 \mathrm{~F}(\mathrm{n}=6)$ & $1 \mathrm{M} / 6 \mathrm{~F}(\mathrm{n}=7)$ & 1 \\
\hline Race & $3 \mathrm{AA} / 3 \mathrm{C}(\mathrm{n}=6)$ & $2 \mathrm{AA} / 4 \mathrm{C} / 1 \mathrm{H}(\mathrm{n}=7)$ & $\mathrm{N} / \mathrm{A}$ \\
\hline Age at CVA dx & $60.2 \pm 14.9(\mathrm{n}=5)$ & N/A & N/A \\
\hline Age at SSc dx & $49.2 \pm 20.1(n=6)$ & $40.3 \pm 9.6(n=7)$ & 0.32 \\
\hline Hypertension & 5 yes $/ 1$ no $(\mathrm{n}=6)$ & 5 yes $/ 2$ no $(n=7)$ & 1 \\
\hline Hyperlipidemia & 3 yes/3 no $(n=6)$ & 1 yes/6 no $(n=7)$ & 0.27 \\
\hline Prior TIA or CVA & 1 yes $/ 4$ no $(\mathrm{n}=5)$ & $\mathrm{N} / \mathrm{A}$ & $\mathrm{N} / \mathrm{A}$ \\
\hline CAD & 3 yes/3 no $(n=6)$ & 0 yes $/ 7$ no $(\mathrm{n}=7)$ & 0.07 \\
\hline Atrial fibrillation & 0 yes $/ 6$ no $(n=6)$ & 0 yes/7 no $(\mathrm{n}=7)$ & 1 \\
\hline Diabetes mellitus & 1 yes $/ 5$ no $(n=6)$ & 2 yes $/ 5$ no $(n=7)$ & 1 \\
\hline Tobacco use & 3 yes $/ 3$ no $(n=6)$ & 2 yes $/ 5$ no $(n=7)$ & 0.59 \\
\hline Family hx of CVA & 1 yes $/ 5$ no $(n=6)$ & 1 yes/6 no $(n=7)$ & 1 \\
\hline Carotid disease & 2 yes $/ 3$ no $(n=5)$ & 0 yes/ 1 no $(\mathrm{n}=1)$ & 1 \\
\hline Anti-nuclear antibody & $4 \operatorname{pos} / 0$ neg $(n=4)$ & $6 \operatorname{pos} / 0$ neg $(n=6)$ & 1 \\
\hline Scl-70 antibody & $0 \operatorname{pos} / 2 \operatorname{neg}(\mathrm{n}=2)$ & $3 \operatorname{pos} / 3 \operatorname{neg}(n=6)$ & 0.46 \\
\hline ACA & $1 \operatorname{pos} / 2 \operatorname{neg}(\mathrm{n}=3)$ & 0 pos $/ 6$ neg $(n=6)$ & 0.33 \\
\hline $\mathrm{Cr}$ at time of CVA & $1.6 \pm 1.0(\mathrm{n}=4)$ & N/A & N/A \\
\hline $\mathrm{Hb}$ at time of CVA & $10.5 \pm 2.45(\mathrm{n}=4)$ & N/A & N/A \\
\hline Alb at time of CVA & $3.7 \pm 0.40(\mathrm{n}=4)$ & N/A & N/A \\
\hline
\end{tabular}

$D c S S c$ diffuse cutaneous systemic sclerosis, $C V A$ cerebrovascular accident, $M$ male, $F$ female, $A A$ African American, $C$ Caucasian, $H$ Hispanic, $N I A$ not applicable, $d x$ diagnosis, $T I A$ transient ischemic attack, $C A D$ coronary artery disease, $h x$ history, pos positive, neg negative, $A C A$ anti-centromere antibody, $C r$ creatinine, $H b$ hemoglobin, $A l b$ albumin. 
Table 3. Comparison of LcSSc patients with CVA to LcSSc patients without a CVA.

\begin{tabular}{|c|c|c|c|}
\hline Parameter & $\mathrm{LcSS} c+\mathrm{CVA}(\mathrm{n}=19)$ & $\mathrm{LcSS} c+$ no CVA $(\mathrm{n}=29)$ & $p$ value \\
\hline Sex & $2 \mathrm{M} / 17 \mathrm{~F}(\mathrm{n}=19)$ & $2 \mathrm{M} / 27 \mathrm{~F}(\mathrm{n}=29)$ & 1 \\
\hline Race & $8 \mathrm{AA} / 11 \mathrm{C}(\mathrm{n}=19)$ & $4 \mathrm{AA} / 25 \mathrm{C}(\mathrm{n}=29)$ & 0.041 \\
\hline Age at CVA dx & $53.5 \pm 14.9(\mathrm{n}=19)$ & N/A & N/A \\
\hline Age at SSc dx & $51.7 \pm 16.0(\mathrm{n}=17)$ & $55.6 \pm 14.5(\mathrm{n}=29)$ & 0.4 \\
\hline Hypertension & 12 yes $/ 7$ no $(n=19)$ & 12 yes $/ 17$ no $(n=29)$ & 0.24 \\
\hline Hyperlipidemia & 9 yes/10 no $(n=19)$ & 15 yes $/ 14$ no $(\mathrm{n}=29)$ & 1 \\
\hline Prior TIA or CVA & 8 yes/11 no $(n=19)$ & N/A & N/A \\
\hline CAD & 4 yes $/ 15$ no $(n=19)$ & 3 yes $/ 26$ no $(n=29)$ & 0.41 \\
\hline Atrial fibrillation & 5 yes $/ 14$ no $(n=19)$ & 2 yes/27 no $(n=29)$ & 0.1 \\
\hline Diabetes mellitus & 2 yes $/ 17$ no $(\mathrm{n}=19)$ & 1 yes $/ 28$ no $(n=29)$ & 0.55 \\
\hline Tobacco use & 10 yes $/ 9$ no $(n=19)$ & 13 yes $/ 16$ no $(n=29)$ & 0.77 \\
\hline Family hx of CVA & 4 yes/15 no $(n=19)$ & 3 yes/26 no $(n=29)$ & 0.41 \\
\hline Carotid disease & 8 yes $/ 6$ no $(n=14)$ & 2 yes $/ 0$ no $(n=2)$ & 0.5 \\
\hline Anti-nuclear antibody & 15 pos $/ 1$ neg $(\mathrm{n}=16)$ & $26 \operatorname{pos} / 3$ neg $(\mathrm{n}=29)$ & 1 \\
\hline Scl-70 antibody & 1 pos/9 neg $(n=10)$ & $1 \operatorname{pos} / 23$ neg $(\mathrm{n}=24)$ & 0.51 \\
\hline $\mathrm{ACA}$ & 8 pos/8 neg $(n=16)$ & 17 pos $/ 10$ neg $(\mathrm{n}=27)$ & 0.53 \\
\hline $\mathrm{Cr}$ at time of CVA & $1.1 \pm 0.60(\mathrm{n}=17)$ & N/A & N/A \\
\hline $\mathrm{Hb}$ at time of CVA & $12.6 \pm 1.40(\mathrm{n}=17)$ & N/A & $\mathrm{N} / \mathrm{A}$ \\
\hline Alb at time of CVA & $3.8 \pm 0.52(\mathrm{n}=16)$ & N/A & N/A \\
\hline
\end{tabular}

$L c S S c$ limited cutaneous systemic sclerosis, $C V A$ cerebrovascular accident, $M$ male, $F$ female, $A A$ African American, $C$ Caucasian, $N / A$ not applicable, $d x$ diagnosis, $T I A$ transient ischemic attack, $C A D$ coronary artery disease, $h x$ history, pos positive, neg negative, $A C A$ anti-centromere antibody, $C r$ creatinine, $H b$ hemoglobin, $A l b$ albumin.

When comparing the LcSSc + CVA to the DcSSc + CVA patients, the DcSSc + CVA patients had higher creatinine levels and lower hemoglobin levels though only the difference in hemoglobin level was statistically significant. Atrial fibrillation was seen in five of the LcSSc + CVA patients but none of the DcSSc + CVA patients, though this did not reach statistical significance (Table 4).

\section{Discussion}

The purpose of this case control study was to identify possible clinical characteristics that might place SSc patients at a greater risk for suffering a CVA. Based on a large cohort of SSc patients $(n=2080)$ followed at our institution between October 2015 and April 2019, we identified 36 SSc patients who developed a subsequent CVA (1.7\% of cohort). When looking at risk factors for CVA in SSc patients, we identified hypertension and atrial fibrillation to be associated with the diagnosis of CVA in such patients. Specifically, 28 of the 36 patients with both SSc and CVA also had a diagnosis of hypertension while in the control 
group only 17 of 36 patients had hypertension $(p=0.014)$. Atrial fibrillation was seen in 9 of 36 patients with both SSc and CVA while it was seen in only 2 of 36 patients in the control group $(p=0.046)$ (Table 1).

Via its contribution to the formation of atherosclerosis, hypertension is regarded as the most important modifiable risk factor for CVA [18]. Similarly, atrial fibrillation is believed to increase the risk of CVA by three to five times in the general population by allowing for the stagnation of blood in the left atrial appendage, thus making one susceptible to thrombus formation and embolism [19]. The relative risk for ischemic stroke attributed to diabetes has been shown to be 2.45 . It is believed that diabetes may hasten the progression of atherosclerosis and is often associated with other risk factors that are themselves risk factors for CVA, such as central obesity, hyperlipidemia, and hypertension [20]. Finally, the relative risk for CVA attributed to smoking has been shown to be 1.5 [21].

Table 4. Comparison of LcSSc patients with CVA to DcSSc patients with CVA.

\begin{tabular}{cccc}
\hline Parameter & LcSSc + CVA $(\mathrm{n}=19)$ & DcSSc + CVA $(\mathrm{n}=6)$ & $p$ value \\
\hline Sex & 2 M/17 F $(\mathrm{n}=19)$ & 1 M/5 F $(\mathrm{n}=6)$ & 1 \\
Race & 8 AA/11 C $(\mathrm{n}=19)$ & 3 AA/3 C $(\mathrm{n}=6)$ & 1 \\
Age at CVA dx & $53.5 \pm 14.9(\mathrm{n}=19)$ & $60.2 \pm 14.9(\mathrm{n}=5)$ & 0.69 \\
Age at SSc dx & $51.7 \pm 16.0(\mathrm{n}=17)$ & $49.2 \pm 20.1(\mathrm{n}=6)$ & 0.76 \\
Hypertension & 12 yes/7 no $(\mathrm{n}=19)$ & 5 yes/1 no $(\mathrm{n}=6)$ & 0.62 \\
Hyperlipidemia & 9 yes/10 no $(\mathrm{n}=19)$ & 3 yes/3 no $(\mathrm{n}=6)$ & 1 \\
Prior TIA or CVA & 8 yes/11 no $(\mathrm{n}=19)$ & 1 yes/4 no $(\mathrm{n}=5)$ & 0.61 \\
CAD & 4 yes/15 no $(\mathrm{n}=19)$ & 3 yes/3 no $(\mathrm{n}=6)$ & 0.3 \\
Atrial fibrillation & 5 yes/14 no $(\mathrm{n}=19)$ & 0 yes/6 no $(\mathrm{n}=6)$ & 0.29 \\
Diabetes mellitus & 2 yes/17 no $(\mathrm{n}=19)$ & 1 yes/5 no $(\mathrm{n}=6)$ & 1 \\
Tobacco use & 10 yes/9 no $(\mathrm{n}=19)$ & 3 yes/3 no $(\mathrm{n}=6)$ & 1 \\
Family hx of CVA & 4 yes/15 no $(\mathrm{n}=19)$ & 1 yes/5 no $(\mathrm{n}=6)$ & 1 \\
Carotid disease & 8 yes/6 no $(\mathrm{n}=14)$ & 2 yes/3 no $(\mathrm{n}=5)$ & 0.63 \\
Anti-nuclear antibody & 15 pos/1 neg $(\mathrm{n}=16)$ & 4 pos/0 neg $(\mathrm{n}=4)$ & 1 \\
Scl-70 antibody & 1 pos/9 neg $(\mathrm{n}=10)$ & 0 pos/2 neg $(\mathrm{n}=2)$ & 1 \\
ACA & 8 pos/8 neg $(\mathrm{n}=16)$ & 1 pos/2 neg $(\mathrm{n}=3)$ & 0.037 \\
Cr at time of CVA & $1.1 \pm 0.60(\mathrm{n}=17)$ & $1.6 \pm 1.0(\mathrm{n}=4)$ & 0.93 \\
Hb at time of CVA & $12.6 \pm 1.40(\mathrm{n}=17)$ & $10.5 \pm 2.45(\mathrm{n}=4)$ & 1 \\
Alb at time of CVA & $3.8 \pm 0.52(\mathrm{n}=16)$ & $3.7 \pm 0.40(\mathrm{n}=4)$ & 1 \\
\hline
\end{tabular}

$L c S S c$ limited cutaneous systemic sclerosis, $D c S S c$ diffuse cutaneous systemic sclerosis, $C V A$ cerebrovascular accident, $M$ male, $F$ female, $A A$ African American, $C$ Caucasian, $N / A$ not applicable, $d x$ diagnosis, $T I A$ transient ischemic attack, $C A D$ coronary artery disease, $h x$ history, pos positive, neg negative, $A C A$ anti-centromere antibody, $C r$ creatinine, $H b$ hemoglobin, $A l b$ albumin. 
Hypertension is not known to be more prevalent in patients with SSc. While previous research has suggested that systemic inflammation may play a role in the pathogenesis of atrial fibrillation [22], there has been insufficient evidence to conclude that atrial fibrillation is more commonly found in autoimmune rheumatic diseases than in the general population. However, it has been reported that the prevalence of atrial fibrillation is higher in SSc than in other autoimmune rheumatic diseases [23]. Interestingly, in our small study, there were 4.5 times the number of patients with atrial fibrillation in the experimental group of patients with CVA than in the control group without CVA. Given the findings of our study and the impact that these two risk factors have for the development of CVA in the general population, they likely have an equal, and perhaps greater, contribution to the development of CVA in SSc patients.

Of note, the creatinine of patients in our experimental group was found to be elevated $(1.23 \pm 0.77 \mathrm{mg} / \mathrm{dl}, \mathrm{n}=26)$. This is consistent with a prior case series in which a majority of SSc patients who later developed a myocardial infarction had a creatinine of greater than $1.3 \mathrm{mg} / \mathrm{dl}$ [7]. It has been reported that approximately half of patients with SSc have clinical evidence of renal disease, including hypertension and/or increased creatinine levels. In addition, it has been hypothesized that those with SSc undergo pathologic renal vascular changes (even in the absence of renal crisis) that could result in rapid renal insufficiency during acute illness [24].

Certainly, the size of this study is one of its limitations. With a larger population, some of the other risk factors that did not reach statistical significance, such as hyperlipidemia, CAD, diabetes mellitus, tobacco use, family history of $\mathrm{CVA}$, and carotid disease, could prove to be statistically significant. In particular, given a $p$ value approaching statistical significance in our small study and the reported link previously suggested [7], we would hope to better describe the relationship between CAD and the development of CVA in SSc patients in future, larger studies. In addition, there is some degree of selection bias which comes from the fact that the study population comes entirely from a single university-based hospital.

\section{Conclusion}

In conclusion, our case control study demonstrated that hypertension and atrial fibrillation had a statistically significant association with the diagnosis of CVA in patients with SSc. This provides additional evidence to our understanding of risk factors contributing to morbidity in SSc. Future, larger studies are needed to further characterize these relationships in order to provide even more valuable information to physicians treating patients with SSc.

\section{Ethics Approval and Consent to Participate}

This study was approved by the Institutional Review Board of the University of Pennsylvania. 


\section{Consent for Publication}

Not applicable.

\section{Availability of Data and Materials}

The datasets used and/or analyzed during the current study are available from the corresponding author on reasonable request.

\section{Funding}

This study did not receive financial resources.

\section{Authors' Contributions}

Both authors contributed equally to write and review the manuscript. Both authors read and approved the final manuscript.

\section{Acknowledgements}

CJI would like to thank Nebojsa Mirkovic of the University of Pennsylvania Data Analytics Center for his help with the acquisition of data used for this study.

\section{Conflicts of Interest}

The authors declare no conflicts of interest regarding the publication of this paper.

\section{References}

[1] Bessant, R., Hingorani, A., Patel, L., MacGregor, A., Isenberg, D.A. and Rahman, A. (2004) Risk of Coronary Heart Disease and Stroke in a Large British Cohort of Patients with Systemic Lupus Erythematosus. Rheumatology (Oxford), 43, 924-929. https://doi.org/10.1093/rheumatology/keh213

[2] Maradit-Kremers, H., Nicola, P.J., Crowson, C.S., Ballman, K.V. and Gabriel, S.E. (2005) Cardiovascular Death in Rheumatoid Arthritis: A Population-Based Study. Arthritis \& Rheumatology, 52, 722-732. https://doi.org/10.1002/art.20878

[3] Shen, J., Wong, K.-T., Cheng, I.T., Shang, Q., Li, E.K., Wong, P., et al. (2017) Increased Prevalence of Coronary Plaque in Patients with Psoriatic Arthritis without Prior Diagnosis of Coronary Artery Disease. Annals of the Rheumatic Diseases, 76, 1237-1244. https://doi.org/10.1136/annrheumdis-2016-210390

[4] Libby, P. (2002) Inflammation in Atherosclerosis. Nature, 420, 868-874. https://doi.org/10.1038/nature01323

[5] Shen, J., Shang, Q. and Tam, L.S. (2016) Targeting Inflammation in the Prevention of Cardiovascular Disease in Patients with Inflammatory Arthritis. Translational Research, 167, 138-151. https://doi.org/10.1016/j.trsl.2015.05.006

[6] Jimenez, S.A. and Derk, C.T. (2004) Following the Molecular Pathways toward an Understanding of the Pathogenesis of Systemic Sclerosis. Annals of Internal Medicine, 140, 37-50. https://doi.org/10.7326/0003-4819-140-1-200401060-00010

[7] Derk, C.T. and Jimenez, S.A. (2007) Acute Myocardial Infarction in Systemic Sclerosis Patients: A Case Series. Clinical Rheumatology, 26, 965-968. https://doi.org/10.1007/s10067-006-0211-8 
[8] Donnan, G.A., Fisher, M., Macleod, M. and Davis, S.M. (2008) Stroke. The Lancet, 371, 1612-1623. https://doi.org/10.1016/S0140-6736(08)60694-7

[9] Auer, R.N. and Sutherland, G.R. (2005) Primary Intracerebral Hemorrhage: Pathophysiology. Canadian Journal of Neurological Sciences, 32, S3-S12.

[10] Thrift, A.G., Dewey, H.M., Macdonell, R.A., McNeil, J.J. and Donnan, G.A. (2001) Incidence of the Major Stroke Subtypes: Initial Findings from the North East Melbourne Stroke Incidence Study (NEMESIS). Stroke, 32, 1732-1738. https://doi.org/10.1161/01.STR.32.8.1732

[11] Esmon, C.T. (2005) The Interactions between Inflammation and Coagulation. British Journal of Haematology, 131, 417-430. https://doi.org/10.1111/j.1365-2141.2005.05753.x

[12] Younger, D.S. (2019) Stroke Due to Vasculitis in Children and Adults. Neurologic Clinics, 37, 279-302. https://doi.org/10.1016/j.ncl.2019.01.004

[13] Holmqvist, M., Simard, J.F., Asplund, K. and Arkema, E.V. (2015) Stroke in Systemic Lupus Erythematosus: A Meta-Analysis of Population-Based Cohort Studies. RMD Open, 1, e000168. https://doi.org/10.1136/rmdopen-2015-000168

[14] Man, A., Zhu, Y., Zhang, Y., Dubreuil, M., Rho, Y.H., Peloquin, C., et al. (2013) The Risk of Cardiovascular Disease in Systemic Sclerosis: A Population-Based Cohort Study. Annals of the Rheumatic Diseases, 72, 1188-1193.

https://doi.org/10.1136/annrheumdis-2012-202007

[15] Chiang, C.H., Liu, C.J., Huang, C.C., Chan, W.L., Huang, P.H., Chen, T.J., et al. (2013) Systemic Sclerosis and Risk of Ischaemic Stroke: A Nationwide Cohort Study. Rheumatology (Oxford), 52, 161-165. https://doi.org/10.1093/rheumatology/kes352

[16] Zöller, B., Li, X., Sundquist, J. and Sundquist, K. (2012) Risk of Subsequent Ischemic and Hemorrhagic Stroke in Patients Hospitalized for Immune-Mediated Diseases: A Nationwide Follow-Up Study from Sweden. BMC Neurology, 12, 41. https://doi.org/10.1186/1471-2377-12-41

[17] Ungprasert, P., Sanguankeo, A. and Upala, S. (2016) Risk of Ischemic Stroke in Patients with Systemic Sclerosis: A Systematic Review and Meta-Analysis. Modern Rheumatology, 26, 128-131. https://doi.org/10.3109/14397595.2015.1056931

[18] O’Donnell, M.J., Chin, S.L., Rangarajan, S., Xavier, D., Liu, L., Zhang, H., et al. (2016) Global and Regional Effects of Potentially Modifiable Risk Factors Associated with Acute Stroke in 32 Countries (Interstroke): A Case-Control Study. The Lancet, 388, 761-775. https://doi.org/10.1016/S0140-6736(16)30506-2

[19] Wolf, P.A., Abbott, R.D. and Kannel, W.B. (1991) Atrial Fibrillation as an Independent Risk Factor for Stroke: The Framingham Study. Stroke, 22, 983-988. https://doi.org/10.1161/01.STR.22.8.983

[20] Burchfiel, C.M., Curb, J.D., Rodriguez, B.L., Abbott, R.D., Chiu, D. and Yano, K. (1994) Glucose Intolerance and 22-Year Stroke Incidence: the Honolulu Heart Program. Stroke, 25, 951-957. https://doi.org/10.1161/01.STR.25.5.951

[21] Shinton, R. and Beevers, G. (1989) Meta-Analysis of Relation between Cigarette Smoking and Stroke. BMJ, 298, 789-794. https://doi.org/10.1136/bmj.298.6676.789

[22] Engelmann, M.D. and Svendsen, J.H. (2005) Inflammation in the Genesis and Perpetuation of Atrial Fibrillation. European Heart Journal, 26, 2083-2092. https://doi.org/10.1093/eurheartj/ehi350

[23] Baek, Y.S., Kim, T.H., Uhm, J.S., Kim, J.Y., Pak, H.N., Lee, M.H., et al. (2016) Prevalence and the Clinical Outcome of Atrial Fibrillation in Patients with Autoimmune Rheumatic Disease. International Journal of Cardiology, 214, 4-9. 
https://doi.org/10.1016/j.ijcard.2016.03.083

[24] Steen, V.D., Syzd, A., Johnson, J.P., Greenburg, A. and Medsger, T.A. (2005) Kidney Disease Other than Renal Crisis in Patients with Diffuse Scleroderma. The Journal of Rheumatology, 32, 649-655. 